\title{
Aplicabilidade do Indicador Técnico MACD à Previsibilidade da Vazão Média Anual do Rio Paraná
}

\author{
David Duarte Cavalcante Pinto ${ }^{1}$ (D), Maria Luciene Dias de Melo ${ }^{1}$ (D), \\ Fabrício Daniel dos Santos Silva ${ }^{1}$ (D), Luiz Carlos Baldicero Molion ${ }^{1}$, Rafaela Lisboa Costa ${ }^{1}$ \\ ${ }^{1}$ Instituto de Ciências Atmosféricas, Universidade Federal de Alagoas, Maceió, AL, Brasil.
}

Recebido em: 12 de Julho de 2020 - Aceito em: 22 de Julho de 2020

\begin{abstract}
Resumo
Este artigo avalia a aplicabilidade e efetividade da ferramenta estatística "Convergência e Divergência de Médias Móveis” (MACD) à previsibilidade das vazões médias anuais do Rio Paraná medidas na cidade de Corrientes-Argentina, com amplitude temporal de 100 anos. Esta técnica é amplamente utilizada no mercado financeiro para previsão de tendências e reversões em valores de commodities e ações, tendo sofrido ajustes para uso nesse estudo. Às médias móveis exponenciais (EMA) do oscilador, atribuíram-se períodos correspondentes a ciclos astronômicos que produzem efeitos na variável climatológica em estudo: o Ciclo solar de Schwabe, de periodicidade de 11,2 anos, o Ciclo Nodal Lunar, de 18,6 anos, e o Ciclo Apsidal Lunar, de 8,85 anos. Há um crescente entendimento científico de que tais parâmetros cíclicos astronômicos exercem influência no clima terrestre, inclusive sobre oscilações oceano-atmosféricas de escala global, como a Oscilação Decadal do Pacífico (ODP) e a El Niño-Oscilação Sul (ENOS), que, por sua vez, sabidamente se relacionam à variável, a qual é resposta das condições de pluviometria que levam águas a dois dos rios mais importantes da bacia do Prata: o Paraná e o Uruguai. Propriedades específicas da MACD obtiveram desempenho considerável na predição de tendências de aumento/diminuição em diversos períodos nessa série centenária de vazões. Encoraja-se a aplicação da MACD a outras variáveis climáticas, como precipitação, PNM e TSM, de modo a efetivá-la como mais uma ferramenta a fornecer esteio às previsões climáticas.
\end{abstract}

Palavras-chave: previsibilidade climática, ciclo solar de Schwabe, ciclos lunares, vazão, econometria.

\section{Applicability of the MACD Technical Indicator to the Predictability of the Annual Average Flow of Paraná River}

\begin{abstract}
This article assesses the applicability and effectiveness of the statistical tool "Convergence and Divergence of Moving Averages" (MACD) to the predictability of the average annual flow of the Paraná River measured in the city of Corrientes-Argentina, with a time span of 100 years. This technique is widely used in the financial market for forecasting trends and reversals in commodities and stock prices. Given the versatile nature of this technique, its parameters were easily adapted to be implemented in this study. The periods assigned to the exponential moving averages (EMA), an inherent parameter of the oscillator, allude to astronomical cycles that produce effects on the climatological variable in the addressed timescale, namely, the Schwabe Solar Cycle, with a periodicity of 11.2 years, the Lunar Nodal Cycle, of 18.6 years, and the Apsidal Lunar Cycle, of 8.85 years. There is a growing scientific understanding that such astronomical cyclical parameters do influence the terrestrial climate, especially via the modulation of ocean-atmospheric oscillations of global scale, such as the Pacific Decadal Oscillation (ODP) and El Niño-Oscilação Sul (ENOS), which are known to be related to the variable in study, which, in turn, is responsive to the rainfall conditions that supply water to two of the most important rivers in the La Plata basin: Paraná and Uruguay. Specific properties of MACD obtained considerable performance in predicting trends of increase/decrease in several periods of that centennial data series of river flow. The use of MACD for other climate variables, such as rainfall, Sea Level Pressure and Sea Surface Temperature, is highly encouraged, in order to consummate it as an additional tool to improve confidence in climate prediction.
\end{abstract}

Keywords: climate predictability, Schwabe solar cycle, lunar cycles, flow rate, econometrics.

Autor de correspondência: David Duarte Cavalcante Pinto, david.duarte@icat.ufal.br. 


\section{Introdução}

É já inconteste a assertiva de que uma série de ciclos de atividade e de parâmetros orbitais astronômicos produzem, em variadas medidas, efeitos nos climas terrestres. Parâmetros na geometria da órbita da Terra, variando em dezenas de milhares de anos, produzem, coletivamente, o que se denomina hoje de Ciclos de Milankovitch, que regem a variabilidade climática terrestre naquela escala temporal, segundo numerosos registros geológicos de mudanças climáticas (Dean, 2000). Estudos conduzidos nas últimas décadas indicam, com robustez estatística, uma relação de vários desses ciclos climáticos com variações nos parâmetros orbitais lunares e na atividade magnética solar (Currie, 1983; Guiot, 1988; Nordemann, 2005; Yndestad, 2006).

Um dos resultados efetivos dos movimentos de plasma no interior do sol é a formação do campo magnético solar, o polar e o toroidal. Sobretudo por força do último e sua interação com os fluxos convectivos solares, glóbulos de campo magnético excepcionalmente intensos são criados e afloram à superfície solar (fotosfera), produzindo o fenômeno das manchas solares, cujas observações começaram a ser registradas em 1611. A análise desses dados permitiu observar periodicidades na atividade solar, sendo mais relevantes, para o escopo deste estudo, as de 11,2 anos (Ciclo de Schwabe), 22 anos (Ciclo de Hale) e 87 anos (Ciclo de Gleissberg) (Roshan, 2013; Correa et al., 2020), que sabidamente encontram respostas em diversas variáveis (paleo-) climatológicas (Solanki et al., 2004; Haigh, 2007; Lovely, 2009; Hathaway, 2010), incluindo na vazão do Rio Paraná e precipitação nos Andes (Mauas et al., 2010).

O ciclo de marés de sizígia e quadratura, identificado há séculos, sofre, consoante demonstrado em literatura recente, modulações entre as escalas interdecadal e bidecadal, nomeadamente causadas pelo Ciclo das Apsides Lunares, com periodicidade de 8,85 anos, e pelo Ciclo Nodal Lunar, com periodicidade de 18,6 anos (Keeling e Whorf, 1997; Ceverny e Shaffer, 2001; Lessa et al., 2001; Haigh et al., 2011). Sua influência no transporte de calor pelas correntes oceânicas e, consequentemente, nas estruturas do clima global, encontra relações de oscilações climáticas, como a Oscilação Decadal do Pacífico (ODP) e El Niño-Oscilação Sul (ENOS), com frações e múltiplos desses ciclos, especialmente seus meio-ciclos, ocorrendo em 4,4 e 9,3 anos, e o múltiplo de 56 anos. No que tange ao mecanismo físico que relaciona os ciclos lunares ao clima, segundo Maksimov e Smirnov (1965), é a variação da força gravitacional promovida por Lua e Sol que alteram a intensidade das correntes oceânicas e marés e provocam variações persistentes na temperatura de superfície do mar (TSM) (Keeling e Whorf, 1997; Ceverny e Shaffer, 2001; Yasuda et al., 2006; Yndestad, 2006; Agosta, 2013; Molion, 2017).
Com respaldo, portanto, nessas variações astronômicas e tendo ciência de que elas de fato promovem influências no clima, surge com naturalidade a proposta de se valer das acuradas séries temporais desses parâmetros a fim de possivelmente contribuir para entender o clima passado e visualizar tendências para o futuro, lapidando as previsões climáticas.

Do ponto de vista pragmático, também se vê como adequada a adoção de uma ferramenta estatística nascida num ambiente que lida com séries temporais de natureza caótica, o mercado financeiro. O indicador/oscilador financeiro "Convergência e Divergência de Médias Móveis" (MACD, na sigla em inglês) foi desenvolvido com o objetivo de antever tendências de alta e baixa em preços de ações em bolsas de valores, mas mostra-se incrivelmente versátil quanto à sua aplicação a séries de dados de distintas escalas temporais e grandezas físicas, bem como quanto aos períodos atribuídos às suas médias móveis exponenciais, que podem, a critério do usuário e seus objetivos, corresponder às periodicidades dos ciclos supracitados. Neste sentido, tem-se por objetivo desta pesquisa avaliar a destreza de tal ferramenta na predição do comportamento de uma variável hidrometeorológica (e, na escala em estudo, também, climatológica), a saber, a vazão média anual do Rio Paraná.

\section{Materiais e Métodos}

\subsection{Dados}

Foi usada uma série temporal de 100 anos, de 1901 a 2000, de vazões do Rio Paraná, com medições realizadas em estação fluviométrica localizada na cidade de Corrientes, Argentina, $900 \mathrm{~km}$ ao norte de sua foz, no rio da Prata, e alguns quilômetros ao norte da cidade de Buenos Aires.

A bacia hidrográfica do Rio Paraná tem a quarta maior vazão do mundo (média de $20.600 \mathrm{~m}^{3} / \mathrm{s}$ ) e a quinta maior área de drenagem $\left(3.100 .000 \mathrm{~km}^{2}\right)$, menor apenas, na América do Sul, que a do rio Amazonas (Mauas et al., 2011). O Rio Paraná tem origem na junção dos rios Paranaíba e Grande, e, de lá, segue em sentido sul, coletando água de territórios de quatro países: Brasil, Bolívia, Paraguai e Argentina. Flui por áreas densamente povoadas, sendo de irrefutável relevância para o abastecimento de residências, comércio e indústrias, assim como para o fornecimento de energia elétrica para Paraguai e Brasil, pois nele se encontra a Usina Hidrelétrica de Itaipu, a segunda maior do mundo. Ademais, é também navegável por embarcações de comércio internacional. Por essas razões, sua vazão tem sido medida continuamente desde o início do século XX.

\subsection{MACD - Convergência e Divergência de Médias Móveis}

Em seu contexto de origem, os indicadores técnicos oferecem uma perspectiva única para se analisar a evolu- 
ção do preço de ações. Tenta capturar o comportamento e, por vezes, a psicologia do investidor. Alguns indicadores, como as médias móveis, são derivados de fórmulas simples e seus mecanismos não são difíceis de entender. Outros, como os estocásticos, contêm fórmulas mais complexas e demandam um estudo mais profundo para se obter um bom entendimento deles (Fernández-Blanco et al., 2008).

O indicador MACD foi desenvolvido por Gerald Appel no final dos anos 1970 e é uma ferramenta de análise técnica que pode ser usada para determinar tendências futuras no mercado financeiro ao usar atividade real do mercado para estabelecer padrões de intensidade e debilidade (Appel, 2006). É um dos mais simples e efetivos indicadores de tendência disponíveis, transformando dois indicadores de tendência (médias móveis) em um oscilador de momentum ao subtrair uma média móvel de período mais longo de uma de período mais curto. Como resultado, a MACD oferece o melhor de dois mundos: acompanhamento de tendência e momentum (Stockcharts, 2020).

\subsubsection{Médias móveis exponenciais}

Uma média móvel é meramente uma média, com a particularidade de movimentar-se. É, portanto, calculada para um certo período no passado, para cada ponto dele. As Médias Móveis Exponenciais (EMA, sigla em inglês) visam reagir mais rápido e precisamente às variações de tendências. Fazem-no ao refinar o método de cálculo de modo a dar um peso bem maior aos preços mais recentes, e tal peso decresce exponencialmente à medida que se volta no tempo ao começo da série. O preço mais recente tem uma importância muito mais alta do que aqueles que o antecedem. Uma EMA pode ser calculada do seguinte modo (Eq. (1)):

$$
E M A_{t}=\frac{2}{n} \times P_{t}+\left(1-\frac{2}{n}\right) E M A_{t-1}
$$

em que $E M A_{t}$ é a média exponencial móvel para o instante $t, n$ é a amplitude do período, e $P_{t}$ é o preço de fechamento do dia. A EMA inicial $\left({ }_{t-1}\right)$ é a média móvel simples para o período $n$ da série (Chong, 2008).

\subsubsection{Cálculo da $M A C D$}

O cerne da MACD é a diferença entre duas médias móveis: uma mais rápida, (que reflete tendências de mercado de curto prazo) menos uma mais lenta (que reflete tendências de longo prazo (Appel, 2006)). Frequentemente, utilizam-se dois diferentes conjuntos de médias móveis para se calcular a MACD: uma EMA de 12 períodos em combinação com uma EMA de 26 períodos. O idealizador da MACD, Gerald Appel, também sugere o par 19-39.
A linha, ou curva, da MACD é obtida pela subtração da EMA mais lenta (e.g. 26) da mais rápida (e.g. 12), isto é (Fernández-Blanco et al., 2008):

$$
M A C D=E M A(12)-E M A(26)
$$

Os preços de encerramento do dia são geralmente usados para essas EMA. Uma terceira EMA, normalmente de 9 períodos, é plotada, de modo a agir como Linha de Sinal (ou de "gatilho") e identificar pontos de inflexão. Essa é uma EMA da própria MACD, não mais da variável original. Esses três parâmetros definem o indicador MACD. No entanto, é frequente também o uso de um quarto, o Histograma da MACD, que representa a diferença entre a MACD e sua EMA de 9 períodos. O Histograma é positivo quando a linha da MACD está acima da Linha de Sinal e negativo quando o inverso ocorre (Stockcharts, 2020). Destarte, pode-se resumir os parâmetros envolvidos numa MACD usual na seguinte relação:

- EMA de 26 períodos

- EMA de 12 períodos

- Linha (ou Curva) da MACD: EMA de 12 menos EMA de 26

- Linha de Sinal (ou de Gatilho): EMA de 9 da MACD

- Histograma da MACD: Linha da MACD menos a Linha de Sinal

\subsubsection{MACD - Padrões e interpretações de sinais}

Duas das mais importantes características da MACD são sua posição acima ou abaixo da Linha Zero e o sinal da derivada da curva, isto é, se ela está subindo ou descendo. Via de regra, o mercado é mais desfavorável quando a MACD está caindo e abaixo de zero, significando que as tendências de mercado de curto prazo estão mais fracas que as de longo prazo (Appel, 2006).

Com respeito aos termos que dão nome à MACD, diz-se que há uma Convergência quando as EMA se movem uma em direção à outra. Divergência ocorre quando as EMA se distanciam uma da outra. A EMA mais curta (12 períodos) é mais rápida e responsável pela maior parte dos movimentos da MACD. A EMA mais longa (26 períodos) é mais lenta e menos reativa às variações de preços (Stockcharts, 2020). Os parâmetros técnicos básicos da MACD estão ilustrados graficamente na Fig. 1.

\subsubsection{MACD - Crossovers de Centro}

Esse tipo de Crossover indica que a EMA 12 cruzou a EMA 26. O sentido que a MACD adquire, claro, depende do sentido do cruzamento entre as EMA. Uma MACD positiva (acima da Linha Central, ou Linha Zero) indica que a EMA 12 está acima da EMA 26. O valor fica cada vez mais positivo à medida que a EMA mais rápida diverge da EMA mais lenta. Isso significa que o momentum de subida está se intensificando. De modo antagônico, valores negativos de MACD (abaixo da Linha Central) 


\section{MACD}

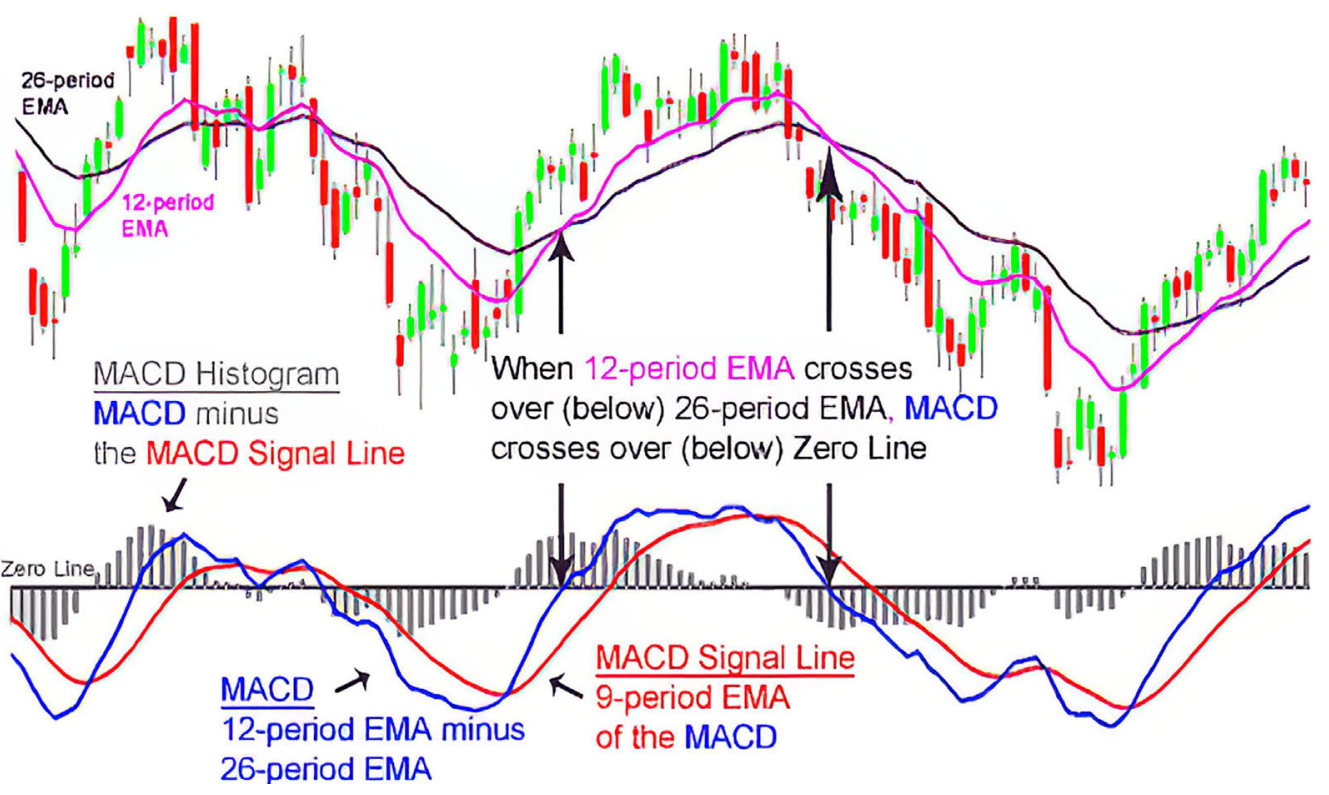

Figura 1 - Parâmetros característicos da MACD. Fonte: Modificado de Wikimedia Commons (2020).

indicam que a EMA 12 está abaixo da EMA 26 e aumentam em valores absolutos à medida que a primeira diverge para baixo da segunda, indicando que o momentum de queda está se intensificando.

Fernández-Blanco et al. (2008) resumem que o cruzar da linha da MACD do lado negativo para o positivo é um sinal de compra (bullish), enquanto o crossover do tipo bearish, i.e., quando a MACD cruza a Linha Central para o lado negativo, é um indicativo de venda.

\subsubsection{MACD - Crossovers da Linha de Sinal}

Fernández-Blanco et al. (2008) asseveram que os principais sinais de compra e venda acontecem quando a curva da MACD intersecta sua própria EMA (a de 9 períodos). Os sinais de compra são gerados quando a linha da MACD cruza a Linha de Sinal de modo ascendente; enquanto a primeira permanecer acima da segunda, devese adotar uma posição de compra. De modo contrário, um sinal de venda ocorre quando a linha da MACD intersecta a Linha de Sinal no sentido descendente; enquanto permanecer abaixo, a posição de venda deve ser mantida.

\subsubsection{MACD - Divergências}

Divergências se formam quando a MACD diverge do comportamento dos preços. Uma divergência bullish se dá quando uma ação denota uma baixa inferior e a MACD exibe uma baixa superior. A baixa inferior afirma a tendência atual de queda, mas a baixa superior na MACD indica um momentum de queda menos intenso. Uma divergência bearish ocorre quando uma ação registra altas superiores e a MACD indica altas inferiores. Uma alta superior no preço de uma ação é normal para uma tendência crescente, mas a baixa inferior da MACD denota menos momentum de subida (Stockcharts, 2020).

\subsubsection{Subidas intensas}

De acordo com Investopedia (2020), subidas dramáticas da MACD - isto é, quando a EMA rápida se distancia sobremaneira da EMA lenta - caracterizam um sinal de sobrecompra (overbuying) da ação, e que logo ela voltará a seu preço normal.

\subsubsection{Prevendo pelo histograma}

O Histograma da MACD foi desenvolvido em 1986 por Thomas Aspray, com vista a reduzir o atraso dos Crossovers da Linha de Sinal. Como já explanado, o Histograma da MACD representa a diferença entre a MACD e sua Linha de Sinal, e é plotado na forma de barras. Notase que, após um crossover, quando o espaço entre a MACD e o Sinal se expande, obtêm-se barras mais longas, que passam a se encurtar conforme as lacunas se reduzem. As barras do Histograma começam a mostrar divergências positivas (bullish) ou negativas (bearish) muito antes do Crossover de Sinal, indicando, assim, antecipadamente, a possibilidade de uma reversão nos preços. Na Fig. 2, observa-se que o Histograma começa a decrescer (barras vermelhas) quando o preço ainda está a subir, bem antes de começar a cair. O sinal do Histograma também antecede bastante o Crossover de Sinal, que só acontece após o 


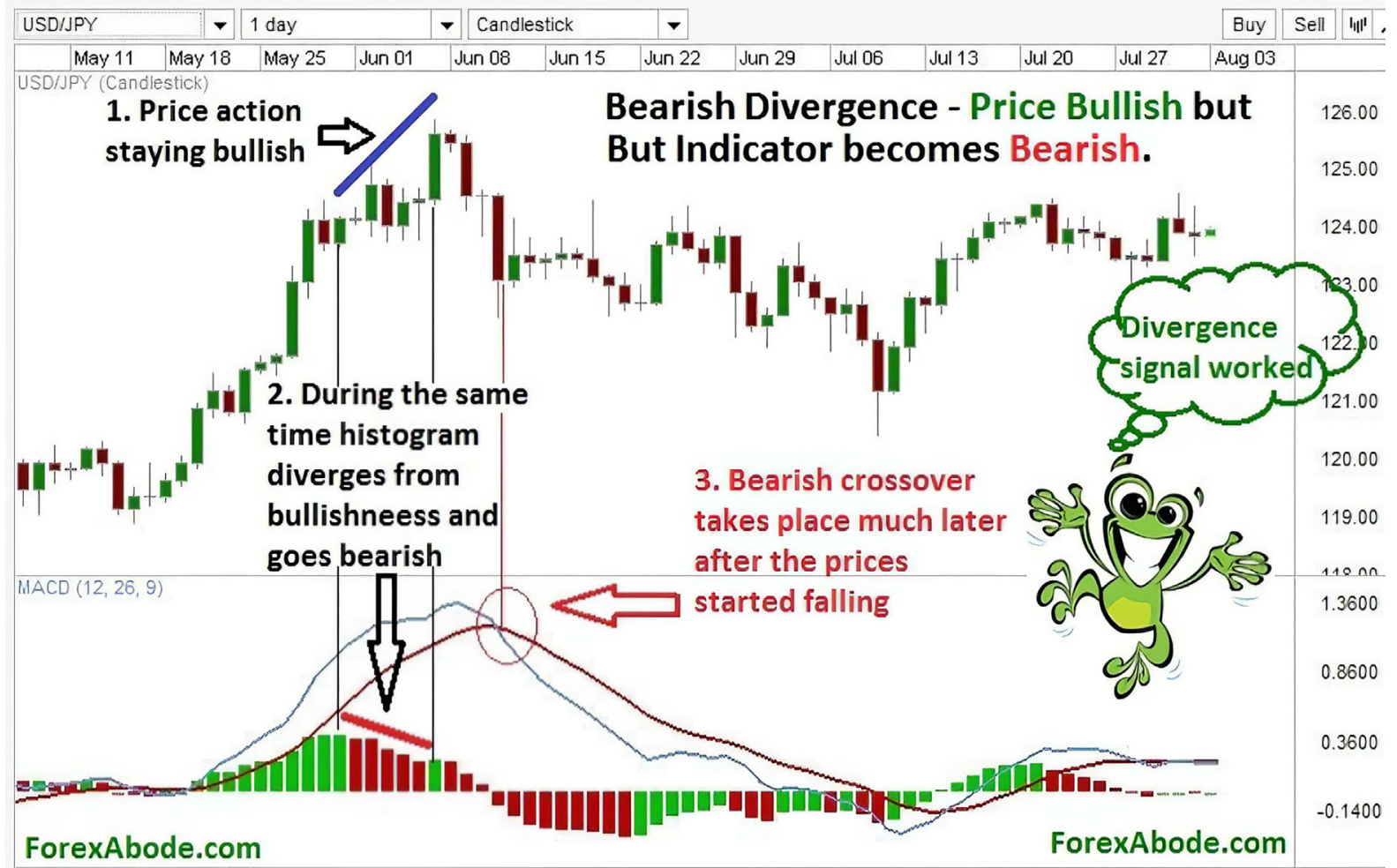

Figura 2 - A Divergência Bearish e a eficácia do Histograma em relação ao Crossover de Sinal. Fonte: ForexAbode (2018).

preço já ter começado sua trajetória de queda. Nesse caso, portanto, o lucro com a venda das ações teria sido maior se o investidor tivesse optado por seguir o indocado pelo Histograma, ao invés do Crossover de Sinal. Todavia, nota-se também que o preço ainda subiu um pouco após o sinal de queda do Histograma. Portanto, sugere-se aguardar algumas barras no Histograma antes de se tomar uma posição (ForexAbode, 2018).

\subsection{MACD - Aplicação prática}

A influência dos parâmetros astronômicos elencados na introdução deste artigo é reconhecida e atestada, em maior ou menor medida, pelos estudos recentes sobre os temas.

A MACD, embora, como já mencionado, tenha berço e finalidade original no mercado financeiro, é uma técnica versátil, inclusive, com relação à escala temporal que se pode empregar. Em que pese seu uso massivo para os valores diários (de fechamento) de preços de ações, valores semanais, mensais e até anuais também são utilizados.

No presente estudo, a técnica viabilizou estabelecer uma relação da variável ora posta em análise com os parâmetros cíclicos astronômicos supracitados, por meio da escolha dos intervalos das EMA. Como visto, a MACD, tradicionalmente, lida com intervalos de 12 períodos para a EMA mais rápida, 26 períodos para a EMA mais longa e 9 períodos para a EMA de Sinal, sendo as duas primeiras calculadas para a variável original e a última calculada sobre a própria MACD.

Aqui, adotaram-se dois outros conjuntos de períodos para o cálculo de cada EMA. A seguir, o primeiro set de períodos, acompanhados da identificação que justifica seu emprego:

- EMA rápida $=11$ anos: Ciclo Solar de Schwabe $(11,2$ anos);

- EMA lenta = 28 anos: Meio Ciclo aproximado da ODP (ciclo total de 50 a 60 anos);

- EMA de Sinal $=9$ anos: Ciclo Lunar das Apsides $(8,85$ anos) ou Meio Ciclo Nodal Lunar (9,3 anos).

Os valores foram arredondados pois a MACD comumente demanda números exatos.

De modo análogo, o segundo conjunto:

- EMA rápida $=9$ anos: Ciclo Lunar das Apsides $(8,85$ anos) ou Meio Ciclo Nodal Lunar (9,3 anos);

- EMA lenta = 19 anos: Ciclo Nodal Lunar (18,6 anos);

- EMA de Sinal = 4 anos: Meio Ciclo Lunar das Apsides (4,4 anos).

Destarte, observa-se que o primeiro conjunto de períodos visa estabelecer uma correspondência das variáveis estudadas com três ciclos distintos, um Solar, outro Lunar e, o terceiro, oceânico, a ODP, embora esta seja influenciada pelo Ciclo Nodal Lunar, tendo uma periodicidade total aproximada de três Ciclos Nodais Lunares (56 anos). Já o segundo é caracterizado pelo uso exclusivo de parâmetros orbitais lunares. 
Para a organização dos dados, cálculos dos parâmetros necessários, como as EMA, a MACD e o Histograma, bem como obtenção das respectivas curvas, fez-se uso do programa gratuito Calc, do pacote LibreOffice (The Document Foundation).

\section{Resultados e Discussão}

Esta seção está organizada como segue: dois gráficos são exibidos para cada conjunto de parâmetros de EMA, o primeiro a conter as curvas da variável original e suas duas EMA e, o segundo, a ilustrar as linhas da MACD, de sua própria EMA (Linha de Sinal) e o Histograma. Posteriormente à exibição dos gráficos, discorre-se devidamente acerca da interpretação dos resultados, à luz do conhecimento científico vigente.

As médias das vazões do Rio Paraná, ao longo de cada ano, na amplitude temporal de 1901 a 2000, são avaliadas quanto à eficiência da previsibilidade da MACD. Nas Figs. 3 e 4, foram aplicados os parâmetros de 11 anos para a EMA rápida, 28 anos para a EMA lenta e 9 anos para a EMA de Sinal, em alusão aos ciclos astronômicos e oceânicos já descritos.

$\mathrm{Na}$ Fig. 3, observa-se, de pronto, que o previsto teoricamente quanto aos sinais demonstrados pelo comportamento das EMA se evidencia. Via de regra, quando as linhas se intersectam, com a EMA de 11 anos cruzando a EMA de 28 para ficar acima, os valores de vazão tendem a aumentar, e o contrário ocorre quando a EMA de 11 anos cruza a EMA de 28 para baixo.

Notam-se poucos Crossovers entre as duas EMA (que são os Crossovers de Centro) ao longo da série, como os que ocorrem nos anos de 1936, 1956, 1963, 1964, 1968 e 1972-73. Dentre eles, os mais eficientes no curto prazo parecem ter sido os de 1936, 1956 e 1964. Já o de 1972-73 sinalizou uma importante tendência de alta de longo prazo até 1984 (12 anos), que é mais bem entendida ao se somar à análise dos outros parâmetros, como Crossover de Sinal e Histograma.

Procedendo à análise, a Fig. 4 denota os outros três parâmetros da MACD, a saber, a Linha da MACD (diferença entre as EMA de 11 e 28 anos), a Linha de Sinal (EMA de 9 anos da MACD) e o Histograma (diferença entre a MACD e a Linha de Sinal). Uma vez que a EMA lenta (de 28 anos) só é calculada e, por conseguinte, plotada, a partir de 1928, e considerando que a MACD é dada pela subtração entre as duas EMA, ela só passa a existir quando há valores para ambas e, portanto, a MACD também só é gerada a partir de 1928 na Fig. 4. De modo análogo, a Linha de Sinal demanda a existência de 9 valores antecedentes da MACD e, portanto, só é gerada a partir de 1936.

O padrão de Crossover de Centro já foi analisado na Fig. 3, pois os Crossovers da Linha Zero, na Fig. 4, ocorrem exatamente quando há um crossover entre as duas EMA na Fig. 3, tendo os mais relevantes já sido apontados. A Fig. 4 permite a análise dos demais padrões e sinais da MACD, que só podem ser avaliados quando é gerada a totalidade dos parâmetros técnicos. Em avaliação dos Crossovers de Sinal, percebe-se que este é mais eficiente na previsão das inversões de tendências de queda ou de alta nos valores de vazão, chegando a preceder, tipicamente, em um a três anos o Crossover de Centro de mesma sinalização.

$\mathrm{Na}$ bolsa de valores, portanto, o Crossover de Sinal denotaria lucros maiores para o investidor do que o Cross-

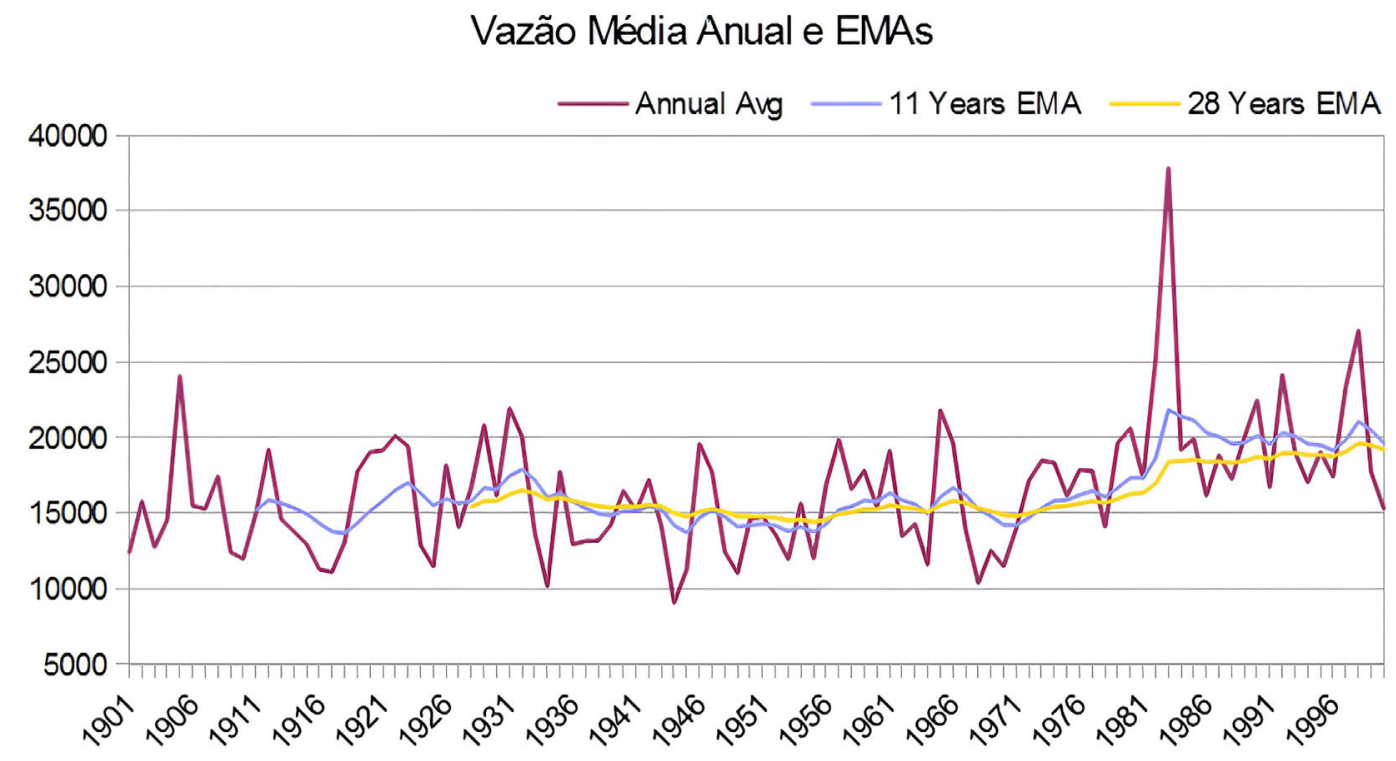

Figura 3 - Curva das médias anuais de vazão do Rio Paraná $\left(\mathrm{em} \mathrm{m}^{3} \cdot \mathrm{s}^{-1}\right)$ segundo medições em Corrientes-ARG no período de 1901 a 2000 , na cor grená. Na mesma escala, também são plotadas, nas cores azul e amarelo, respectivamente, as EMA rápida (11 anos) e lenta (28 anos). 


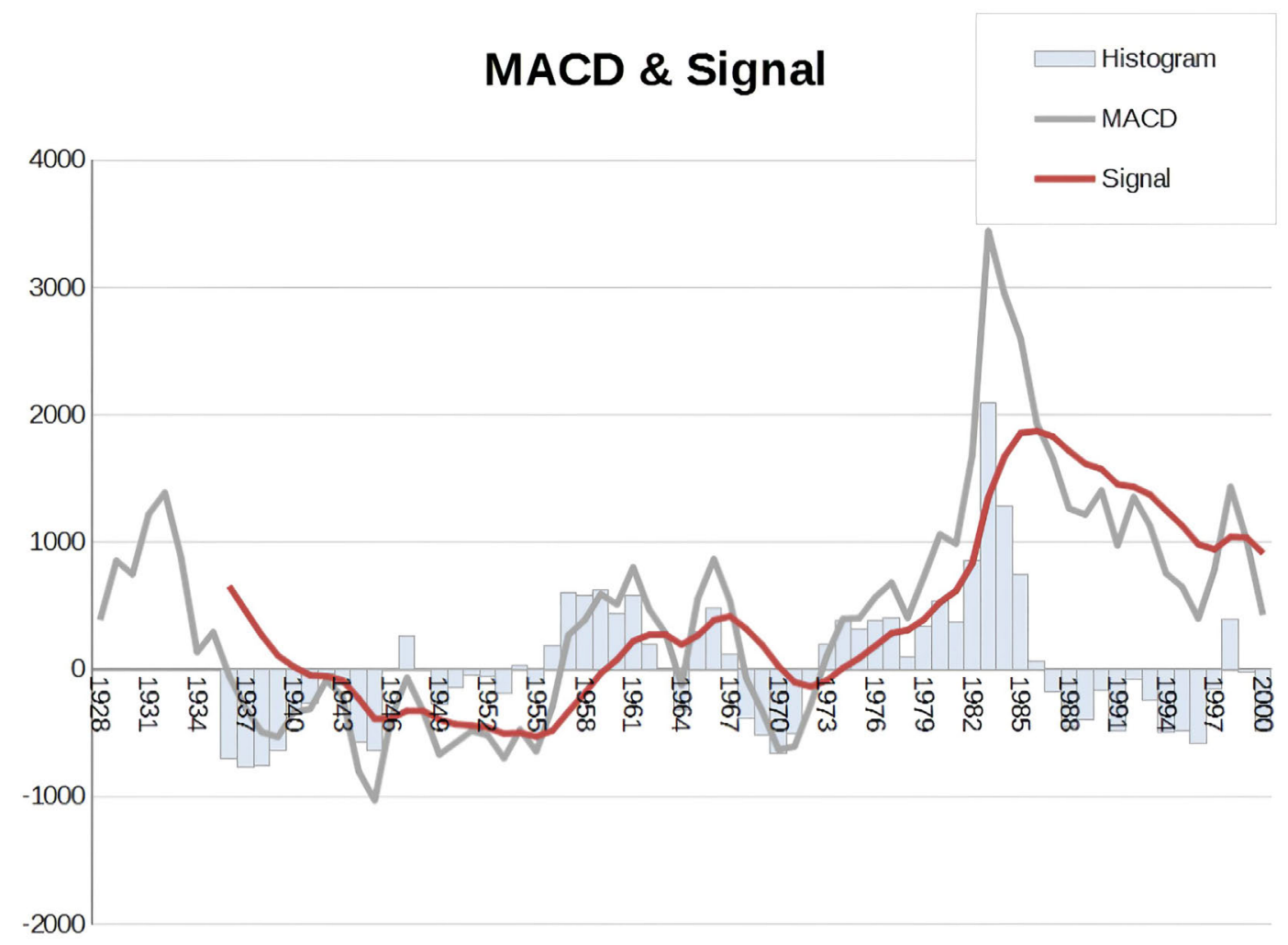

Figura 4 - Linhas da MACD (cinza) e de Sinal (vermelha) para as médias anuais de vazão do Rio Paraná, com parâmetros de EMA de 11, 28 e 9 anos. O Histograma da MACD (colunas azuis) também é exibido.

over de Centro. No presente estudo, significa a relevante utilidade de se prever com mais antecedência e efetividade a variabilidade climatológica associada ao comportamento da variável em estudo.

Acerca das divergências, subidas intensas e previsões pelo Histograma, percebe-se que essas características podem viabilizar uma previsão com antecedência ainda maior que o Crossover de Sinal, embora ofereçam maior risco. Inicialmente, nota-se que as subidas (e descidas) intensas, também vislumbradas na Fig. 3 - quando de divergências acentuadas entre as duas EMA - de fato precedem muitos Crossovers de Sinal e de Centro. A interpretação é que, se o valor está subindo (descendo) demais, logo chegará a um máximo (mínimo), e tenderá a voltar a valores médios, ou seja, uma inversão de comportamento está por acontecer, o que também pode ser entendido pelo fato de que uma divergência acentuada entre as duas EMA implica naturalmente num comportamento bem distinto entre elas, de modo que, contrariamente à mais rápida, a mais lenta tende a denotar cada vez mais precariamente o comportamento atual da variável. Na Fig. 4, o Histograma auxilia na percepção dessas alterações e mesmo a conferir mais segurança na previsão de reversões que anteveem um Crossover de Sinal.

Como esperado, os Crossovers de Sinal precederam a maior parte dos de Centro e sinalizaram importantes al- terações não acusadas pelos primeiros, como em $1972 \mathrm{e}$ 1986. É digno de nota também o Crossover de Sinal de 1955, que indicou uma tendência de aumento de vazão um ano antes do Crossover de Centro.

O Histograma exerce sua relevante função de antever variações não previstas pelos Crossovers, ou antecedêlos em certa medida. Um aumento de vazão que apenas foi ensaiado por um Crossover de Sinal em 1943 fora previsto pelo Histograma em 1939, e a queda subsequente foi antevista em 1944. A Linha de Sinal mostra a tendência de queda das vazões desde seu início e o Crossover bullish em 1955 mostra a mudança da tendência de baixa para alta. Um novo Crossover bullish ocorre em 1972, apontando para uma tendência de alta nas vazões que persistiu até 1984, quando ocorre um Crossover bearish que indica a tendência de queda das vazões até o final da série. Os anos atípicos de 1983 e 1998 são anos de eventos El Niño, em que os totais pluviométricos são excepcionalmente acima da média de longo termo e são semelhantes às "altas inesperadas" no mercado financeiro quando do anúncio de resultados positivos de uma dada empresa num dado trimestre.

Nas Figs. 5 e 6, agora com os intervalos de EMA exclusivamente referentes aos Ciclos Lunares, nas periodicidades de 9 anos para a EMA rápida, 19 anos para a lenta e 4 anos para a de Sinal, percebe-se que a Linha de Sinal 


\section{Vazão Média Anual e EMAs}

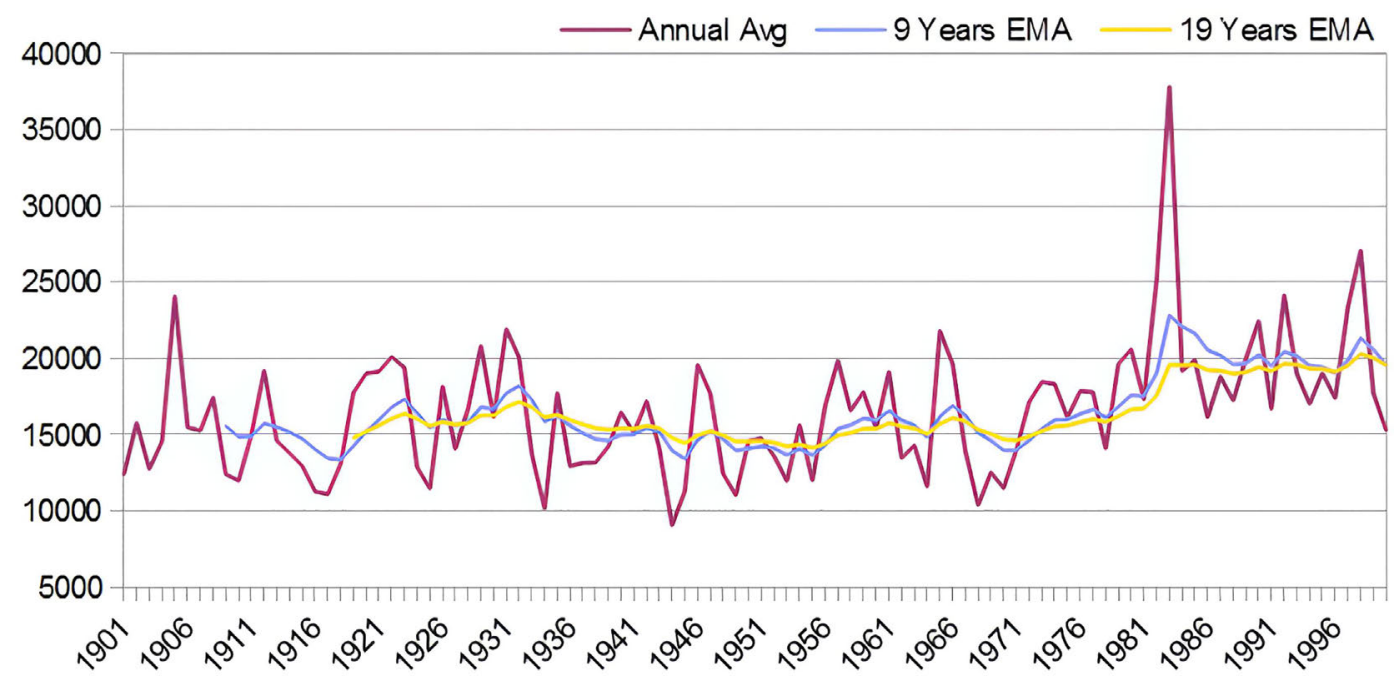

Figura 5 - Curva das médias anuais de vazão do Rio Paraná $\left(\mathrm{em} \mathrm{m}^{3} \cdot \mathrm{s}^{-1}\right)$ segundo medições em Corrientes-ARG no período de 1901 a 2000 , na cor grená. Na mesma escala, também são plotadas, nas cores azul e amarelo, respectivamente, as EMA rápida ( 9 anos) e lenta (19 anos).

\section{MACD \& Signal}

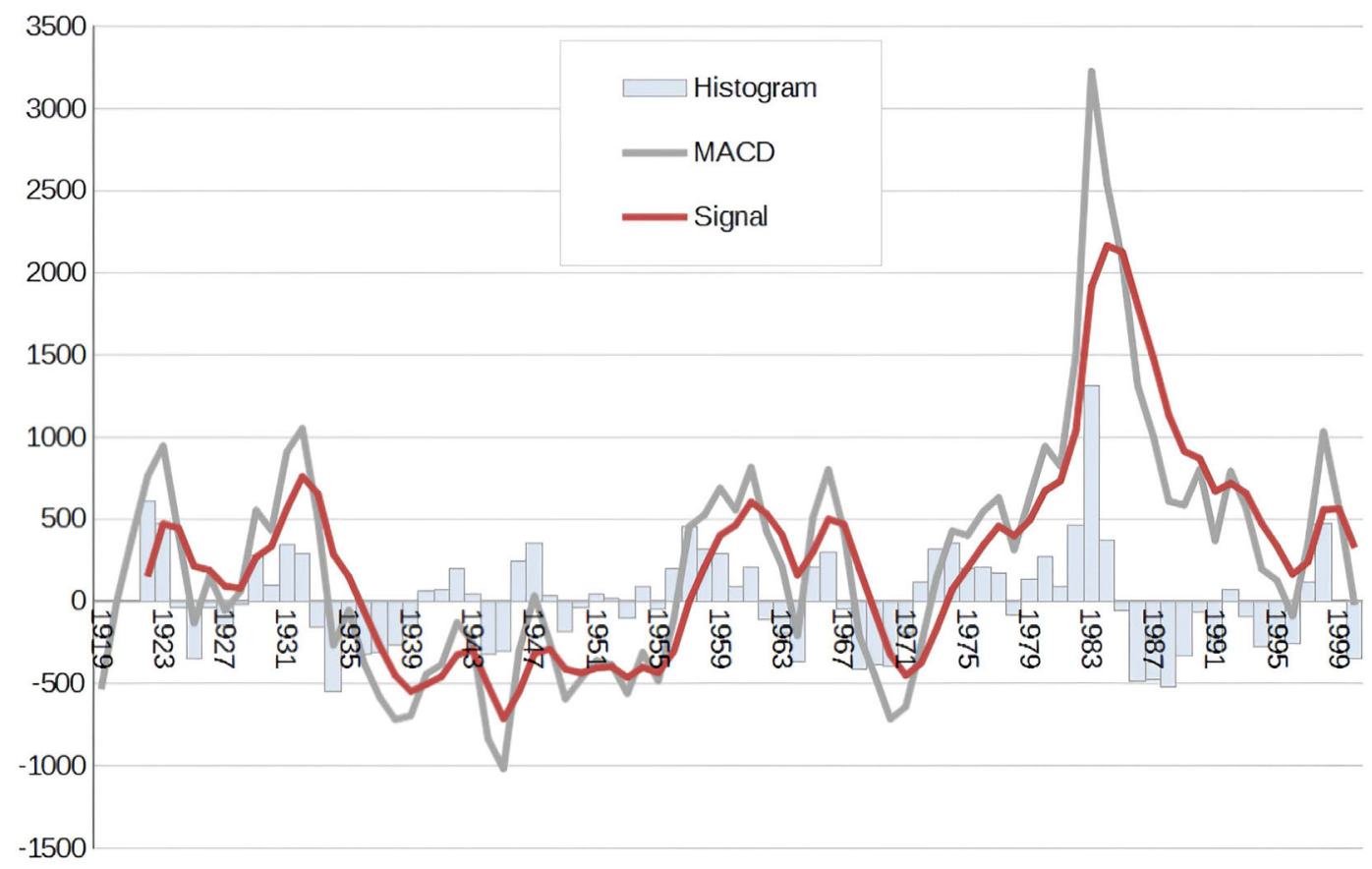

Figura 6 - Linhas da MACD (cinza) e Sinal (vermelha) para as médias anuais de vazão do Rio Paraná, com parâmetros de EMA de 9,19 e 4 anos. O Histograma da MACD (colunas azuis) também é exibido.

acompanha a Linha da MACD bem mais proximamente, ocasionando um número maior de Crossovers de Sinal e um Histograma menos preciso.

Os Crossovers de Centro são praticamente simultâneos em relação aos intervalos de EMA anteriores, ocor- rendo novos que haviam sido meramente ensaiados naqueles, como em 1934, 1947 e 1996. Alguns Crossovers de Sinal tiveram a notável eficácia de anteceder os cruzamentos de mesmo tipo do conjunto de intervalos anteriores em até 2 anos, como em 1984-85, que, na série de 
11-28-9, só ocorre em 1986; em 1971, que lá só se expressa em fins de 1972, e os de 1939 e 1943, que lá foram apenas esboçados. Tais precedências dos Crossovers de Sinal neste conjunto de parâmetros de EMA "lunares" compensam, em certa medida, a maior imprecisão de seu Histograma. O Crossover bearish de 1933 sinaliza uma tendência de baixa das vazões até 1955 (22 anos) quando o Crossover bullish de 1955 indica o início de um período de tendência de oscilação entre vazões altas e baixas. São notórios o Crossover bullish de 1972, que marca o início da tendência de alta das vazões, e o Crossover bearish em 1985, que sinaliza a tendência de queda das vazões que persistiu até o fim da série. Novamente, os valores positivos mais elevados do Histograma são referentes aos eventos El Niño como, por exemplo, 1942, 1946, 1957, 1973, 1983 e 1997.

Por fim, nota-se uma aptidão notória da variável vazão a previsões de médio e longo prazo segundo os parâmetros da MACD. No conjunto de intervalos 11-28-9, especificamente, a tendência de alta da vazão prevista em 1972-73 pelos Crossovers de Centro e de Sinal e indicados em 1971 pelo Histograma, não somente se concretizou, mas subsistiu por quase duas décadas, partindo de valores abaixo de $15.000 \mathrm{~m}^{3} . \mathrm{s}^{-1} \mathrm{e}$ atingindo o pronunciado valor de quase $38.000 \mathrm{~m}^{3} . \mathrm{s}^{-1}$ em 1983, associado ao evento El Niño, que causou impactos mais severos no Sudeste do Brasil.

\section{Conclusões}

A aplicação do oscilador financeiro MACD, como ferramenta analítica, à série histórica hidrometeorológica estudada, provou ser adequada para a tomada de decisão na gestão dos recursos hídricos. Há uma disparidade de eficácias entre os diversos componentes da técnica quanto à antevisão dos sinais de tendência de alta, baixa e suas reversões. Em geral, os Crossovers de Centro são mais lentos, seguidos dos Crossovers de Sinal e do Histograma, que se mostrou o mais célere dos parâmetros. Este, no entanto, apresenta riscos mais altos nas interpretações das tendências futuras da variável, particularmente quando são tomados apenas um ou dois valores, podendo induzir o analista a um eventual equívoco. Pondera-se, portanto, que a MACD se faz muito mais efetiva se utilizada na plenitude de seus parâmetros, com análise criteriosa da relevância de cada um deles, mas sem se tomar decisões com base em apenas um isoladamente.

$\mathrm{Na}$ série de vazões em escrutínio neste trabalho, a MACD foi capaz de antever tendências que durariam até pouco mais de duas décadas. Uma razão para tal comportamento é que a vazão de um rio cuja bacia tem dimensões descomunais, como a do Paraná, integra uma área que coleta precipitações de diversas regiões, tendo as respostas naturalmente suavizadas. Em princípio, parece que a aplicação da MACD a dados fluviométricos em grandes ba- cias resulta em previsões de longo prazo mais confiáveis do que poderia ser sua aplicação a totais pluviométricos.

Com relação aos conjuntos de parâmetros selecionados para gerar as EMA, os períodos referentes exclusivamente aos ciclos orbitais lunares, nomeadamente o Ciclo Lunar das Apsides (8,85 anos = EMA de 9 anos), o Ciclo Nodal Lunar (18,6 anos = EMA de 19 anos) e o Meio Ciclo das Apsides (4,4 anos = EMA de Sinal de 4 anos), mostraram-se bem mais sensíveis a variações de frequência maior, sendo, usualmente, mais eficientes nas previsões de curto prazo, principalmente pelos Crossovers de Sinal. O conjunto de intervalos "mistos", abrangendo o Ciclo Solar de Schwabe (11,2 anos = EMA de 11 anos), o Meio Ciclo da ODP (28 anos = EMA de 28 anos) e o Meio Ciclo Nodal Lunar (9,3 anos = EMA de Sinal de 9 anos) provou ser mais eficaz para previsões de tendências de médio e até de longo prazo no caso das vazões, pois filtra variações provenientes da variabilidade de alta frequência. É aparente que a escolha dos parâmetros para as EMA vai depender do objetivo do trabalho. Por exemplo, se o objetivo é uma análise de tendência de prazo mais longo, utiliza-se a EMA de 9 anos para a MACD, pois ela filtra ou reduz o impacto dos eventos El Niño severos que ocorrem a cada 3-7 anos na série de dados. Mas, se o objetivo é exatamente capturar os impactos dos eventos El Niño na variável, o intervalo de 4 anos é possivelmente o mais indicado para a EMA da MACD (Linha de Sinal).

Assim, por tudo o que precede, as análises conduzidas sinalizam um futuro promissor para o indicador/oscilador MACD na previsibilidade climatológica de séries de vazões e, eventualmente outras variáveis em ciências atmosféricas, apresentando-se apta a municiar os pesquisadores e profissionais da área com mais uma ferramenta, que se provou estatisticamente efetiva e versátil, viabilizando a implementação de variados conjuntos de intervalos em referência a ciclos e índices de oscilações que sabidamente exercem modulações nas variáveis climatológicas que influenciam as regiões estudadas.

\section{Agradecimentos}

Os autores agradecem a Coordenação de Aperfeiçoamento de Pessoal de Nível Superior (CAPES) pelo apoio financeiro concedido durante e para a realização deste estudo.

\section{Referências}

AGOSTA, E.A. The 18.6-year nodal tidal cycle and the bi-decadal precipitation oscillation over the plains to the east of subtropical Andes, South America. International Journal of Climatology, v. 34, p. 1606-1614, 2013.

APPEL, G. Become Your own technical analyst How to Identify Significant Market Turning Points Using the Moving Average Convergence-Divergence Indicator or MACD. The Journal of Wealth Management, v. 6, p. 27-36, 2006. 
CERVENY, R.S.; SHAFFER, J.A. The Moon and El Niño, Geophysical Research Letters, v. 28, p. 25-28, 2001.

CORREA, C.S.; GUEDES, R.L.; ROCHA, A.M.M.; CORREA, K.A.B. Multidecadal Cycles of the Climatic Index Atlantic Meridional Mode: Sunspots that affect North and Northeast of Brazil. Journal of Aerospace Technology and Management, v. 12, e0420, 2020.

CURRIE, R.G. Detection of 18.6-year nodal-induced drought in the Patagonian Andes. Geophysical Research letters, v. 10, p. 1089-1092, 1983.

DEAN, W.E. The Sun and Climate. USGS: Science for a Changing World. Disponível em: http://pubs.usgs.gov/fs/ fs-0095-00/fs-0095-00.pdf. Acesso em: julho de 2020.

FERNÁNDEZ-BLANCO, P.; BODAS-SAGI, D.J.; SOLTERO, F.; HIDALGO, J.I. Technical market indicators optimization using evolutionary algorithms. In: GECCO 08: Proceedings of the 10th Annual Conference Companion on Genetic and Evolutionary Computation, p. 1851-1858, 2008. https://doi.org/10.1145/1388969.1388989.

FOREXABODE. 4.30 - Trading with MACD indicator. Disponível em: http://www.forexabode.com/forex-school/ technical-indicators/macd/. Acesso em: maio de 2018.

GUIOT, J. Climate of the Hudson Bay region and effects of solar activity, volcanic dust and lunar nodal tidal signal. In: CASTAGNOLI C.G. (ed.) Solar-Terrestrial Relationships and the Earth Environment in the Last Millennia. Amsterdam: North Holland, p. 377-395, 1988.

HAIGH, I.D.; ELIOT, M.; PATTIARATCHI, C. Global influences of the 18.61 year nodal cycle and 8.85 year cycle of lunar perigee on high tidal levels. Journal of Geophysical Research-Oceans, v. 116, C06025, 2011.

HATHAWAY, D.H. "The Solar Cycle", Living Rev. Solar Phys., 7:1, 2010.

KEELING, C.D.; WHORF, T.P. Possible forcing of global temperature by the oceanic tides. Proceedings of the National Academy of Sciences, v. 94, p. 8321-8328, 1997.

LESSA, G.C.; DOMINGUEZ, J.M.L.; BITTENCOURT, A.C.S.P.; BRICHTA, A. The tides and tidal circulation of Todos os Santos Bay, Northeast Brazil: a general characterization. Anais da Academia Brasileira de Ciências, v. 73, n. 2, p. 245-261, 2001.

LOVELY, M.R., Study of Dynamics and Magnetic Field Structure of the Solar Convective Envelope using Sun- spot Activity. Tese de Doutorado, Indian Institute of Astrophysics, Bangalore, India, 2009. Disponível em: http://shodhganga.inflibnet.ac.in/bitstream/10603/3912/11/ 11_chapter\%201.pdf. Acesso em: agosto de 2015.

MAKSIMOV, I.V.; SMIRNOV, N.P. A contribution to the study of causes of long period variations in the activity of the Gulf Stream. Oceanology, v. 5, p. 15-24, 1965.

MOLION, L.C.B. Gênese do El Niño, Revista Brasileira de Climatologia, v. 21, p. 2237-8642, 2017.

MAUAS, P.J.D.; BUCCINO, A.P.; FLAMENCO, E. Long-term solar activity influences on South American rivers. Journal of Atmospheric and Solar-Terrestrial Physics, v. 73, n. 2-3, p. 377-382, 2011.

NORDEMANN, D.J.R.; RIGOZOA, N.R.; DE FARIA, H.H. Solar activity and El-Niño signals observed in Brazil and Chile tree ring records. Advances in Space Research, v. 35, n. 5, p. 891-896, 2005.

ROSHAN, G. The relation between the number of sunspots and fluctuation in the trend of climatic components in Iran during recent decades. Environmental Resources Research, v.1, n. 1, p. 65-87, 2013.

SOLANKI, S.K.; USOSKIN, I.G.; KROMER, B.; SHÜSSLER, M.; BEER, J. Unusual activity of the Sun during recent decades compared to the previous 11,000 years. Nature, v. 431, p. 1084-1087, 2004.

STOCKCHARTS, Moving Average Convergence/Divergence Oscillator (MACD). Disponível em: http://stockcharts. com/school/doku.php?id=chart_school:technical_indicators:moving_average_convergence_divergence_macd. Acesso em: junho de 2020.

YASUDA, I.; OSAFUNE, S.; TATEBE, H. Possible explanation linking 18.6-year period nodal tidal cycle with bi-decadal variations of ocean and climate in the North Pacific. Geophysical Research Letters, v. 33, L08606, 2006.

YNDESTAD, H. The influence of the lunar nodal cycle on Arctic climate. ICES Journal of Marine Science, v. 63, n. 3, p. 401-420, 2006.

License information: This is an open-access article distributed under the terms of the Creative Commons Attribution License (type CC-BY), which permits unrestricted use, distribution and reproduction in any medium, provided the original article is properly cited. 